\title{
Molecular regulation of T-ALL cell infiltration into the CNS
}

\author{
Judy L. Cannon, Sreenivasa Rao Oruganti and Devan W. Vidrine
}

Acute lymphoblastic leukemia (ALL) is a blood disorder arising from cells derived from either the T- or B-cell lineage. ALL is the most commonly seen cancer in pediatric patients, with T-cell acute lymphoblastic leukemia (T-ALL) accounting for nearly $20 \%$ of newly diagnosed ALL cases. Advances in therapy are improving event-free survival rates of T-ALL which exceed $80 \%$ in some clinical trials. However, central nervous system (CNS) infiltration by T-ALL cells and CNS relapse in pediatric T-ALL patients drive down survival rates, in some cases to as low as $40 \%$. Current clinical practice addresses T-ALL CNS involvement with high-dose cranial radiation and intrathecal chemotherapy, resulting in adverse effects including neurotoxicity and secondary neoplasms. Successful management of CNS-implicated T-ALL disease remains problematic, largely because homing and survival mechanisms of leukemic cells into the CNS are unclear. Further, exactly which biomarkers drive CNS T-ALL disease remain unidentified. Current research efforts, including our own, seek potential molecular targets for blocking T-ALL disease in the CNS [1].

T-ALL arises from aberrant activation of a variety of signaling pathways that mediate cell growth, survival, and differentiation of thymocytes [2]. Notch1 is a major transcription factor driving T-cell development, and accumulated mutations leading to constitutive activation of the Notch1 pathway are linked to more than half of T-ALL cases. Animal models using oncogenic Notch1 expression to investigate mechanisms of T-ALL disease have been useful to understand the molecular basis of T-ALL, providing therapeutic targets [1, 3]. Recent findings suggest molecules crucial for T-cell receptor (TCR) signaling, like ZAP70 and PKC $\theta$, can play a role in T-ALL pathogenesis [4]. Downstream of ZAP70 and PKC $\theta$, TCR signaling activates Carma1, Bcl10, and MALT1, collectively known as the CBM complex, resulting in NF- $\kappa \mathrm{B}$ activation [5]. Constitutive activation of CBM signaling is now recognized as a common feature of an increasing number of B- and T-cell malignancies [5].

We tested the role of Carmal in T-ALL leukemogenesis and found that Carma1 regulates T-ALL CNS disease [1]. Absence of Carmal leads to increased survival in T-ALL using multiple animal models of T-ALL. Strikingly, Carma1 expression controls migration of T-ALL cells into the CNS. Histological examination of CNS tissue in animals with Carma1-deficient T-ALL cells shows little to no signs of leukemic cell infiltration into the
CNS. This is in contrast to WT T-ALL cells which show significant accumulation in the CNS. Further, in agreement with existing data that correlates increased expression of Carmal with T-ALL disease, we find that CNS-positive T-ALL patients express significantly higher Carmal in comparison with T-ALL patients that are CNS-negative at the time of diagnosis [1]. Our study highlights the essential role of Carma1 in T-ALL disease, particularly in driving CNS infiltration of T-ALL cells. These results suggest Carmal inhibition is a potential therapeutic strategy to control T-ALL CNS disease.

In addition to Carma1, other groups have demonstrated a role for the chemokine receptor CCR7 in trafficking of leukemic T-cells into the CNS [3]. CCR7 typically mediates naïve T-cell migration to lymph nodes, yet its presence on T-ALL cells decreases survival of animals with T-ALL. Interestingly, our research finds that CCR7 and Carma1 co-localize in T-ALL cells, and Carmal expression is important in migration of leukemic T-cells in response to the CCR7 ligand CCL21 [1]. While our data suggests that CCR7 may be upstream of Carma1 in driving T-ALL cell migration to the CNS, it is also possible that CCR7 and Carma1 regulate T-ALL cell migration independently of one another. Besides CCR7, other chemokine signaling pathways including CXCR3 and CXCR4 have been shown to play a role in initiation and progression of T-ALL disease $[3,6,7]$. How CCR7 and other chemokine receptors interact with signaling pathways such as Carmal in regulating CNS disease in T-ALL remains to be clarified.

Movement of immune cells through the blood-brain barrier into the CNS is a decades-old topic of interest, yet much is left to discover. Identifying molecules that drive leukemic T-cells to the CNS has implications not only for T-ALL, but diseases like multiple sclerosis, in which lymphocyte trafficking to the CNS is a central issue. Several targetable pathways including Notch1, IL-7/IL7R, PI3K/Akt, and MAPK are associated with T-ALL progression [8], but the role for these pathways in T-ALL CNS migration thus far remains unexplored. Future investigations into molecular determinants of T-ALL CNS migration hold promise for developing novel therapies to specifically target T-ALL CNS disease.

Judy L. Cannon: Department of Molecular Genetics and Microbiology \& Department of Pathology University of New Mexico Health Sciences Center, Albuquerque, New Mexico, USA 
Correspondence to: Judy L. Cannon, email jucannon@ salud.unm.edu

Keywords: T-ALL, chemokine, carmal, CNS

Received: August 29, 2017

Published: September 30, 2017

\section{REFERENCES}

1. Oruganti SR, et al. Leukemia. 2017; 31:255-258.

2. Belver L, et al. Nat Rev Cancer. 2016; 16:494-507.

3. Buonamici S, et al. Nature. 2009: 459:1000-1004.

4. Felli MP, et al. Oncogene. 2005; 24:992-1000.

5. Thome M, et al. Cold Spring Harb Perspect Biol. 2010; 2: a003004.

6. Gomez AM, et al. Blood Cells Mol Dis. 2015; 55:220-227.

7. de Bock CE, et al. Cancer Cell. 2015; 27:745-746.

8. Silva A, et al. Cancer Res. 2011; 71:4780-4789.

Copyright: Cannon et al. This is an open-access article distributed under the terms of the Creative Commons Attribution License 3.0 (CC BY 3.0), which permits unrestricted use, distribution, and reproduction in any medium, provided the original author and source are credited. 